\title{
2-(2,6-二甲基苯胺基)-2-氧代乙酸促进的铜催化的芳基溴代物的羟基化
}

\author{
刘向前 $a$ 张 慧 $*, a$ 蒋咏文 $*, b$ \\ $\left({ }^{a}\right.$ 上海大学化学系 上海 200444) \\ $\left({ }^{b}\right.$ 中国科学院上海有机化学研究所 生命有机国家重点实验室 上海 200032)
}

\begin{abstract}
摘要 $\mathrm{CuI} / 2$-(2,6-二甲基苯胺基)-2-氧代乙酸作为一个高效的催化体系被成功用于芳基溴代物的羟基化反应. 在此催 化体系下，催化剂用量降至 $2 \mathrm{~mol} \%$, 可用于合成各类取代苯酚. 通过调整反应条件，该催化体系还可用于促进芳香溴 代物一锅法合成对称的芳香醚.
\end{abstract}

关键词＼cjkstart铜; Ullmann 反应; 2-(2,6-二甲基苯胺基)-2-氧代乙酸; 差基化; 酚

\section{2-(2,6-Dimethylphenylamino)-2-oxoacetic Acid as an Efficient Prompter for the Copper-Catalyzed Hydroxylation of Aryl Bromides}

\author{
Liu, Xiangqian ${ }^{a} \quad$ Zhang, Hui $^{*, a} \quad$ Jiang, Yongwen ${ }^{*, b}$ \\ $\left({ }^{a}\right.$ Department of Chemistry, Shanghai University, Shanghai 200444) \\ ( ${ }^{b}$ State Key Laboratory of Bioorganic and Natural Products Chemistry, Shanghai Institute of Organic Chemistry, \\ Chinese Academy of Sciences, Shanghai 200032)
}

\begin{abstract}
CuI/2-(2,6-dimethylphenylamino)-2-oxoacetic acid (DMPAO) as an efficient catalytic combination was used for the hydroxylation of aryl bromides. The catalyst loading is low to $2 \mathrm{~mol} \%$ and substituted phenols could be prepared in wide assembly. In addition, by modifying the mixed solvent and base the symmetrical diaryl ethers could be synthesized conveniently from aryl bromides.

Keywords copper; Ullmann reaction; 2-(2,6-dimethylphenylamino)-2-oxoacetic acid; hydroxylation, phenol
\end{abstract}

最近的研究发现,一些特殊配体在铜催化的卤苯羟 基化反应中起到关键的作用，这为制备取代的苯酚提供 了一种简单实用的方法. 文献中已经报道的有效配体包 括二苯甲酰甲烷 ${ }^{[1]}, N, N$-二甲基乙二胺(DMEDA $)^{[1]}$, 1,10-菲罗啉 ${ }^{[2]}, 8$-羞基喹啉 ${ }^{[3]}, 2$-吡啶甲醛肜 ${ }^{[4]}, 8$-差基喹 哪啶 ${ }^{[5]}, 8$-差基喹啉- $N$-氧化物 ${ }^{[6]}$, 哌啶-2-甲酸锂 ${ }^{[7]}$ 和 $D$ 葡萄糖 ${ }^{8]}$. 这些配体与铜盐组成的催化体系都能高效地 在温和的条件下实现芳基碘代物的羟基化，但对于较不 活泼的芳基溴代物则需要活化溴代物或者采用较为剧 烈的反应条件才能促使其完全转化. 如 Taillefer 小组 ${ }^{[1]}$ 借助碘卤交换的关键过程首次实现了铜催化的芳基溴 代物的差基化. 提高反应温度或增加铜盐和配体的用量 也是促进芳基澳代物的羟基化的常用方法. 如
Leadbeater 等 ${ }^{[9]}$ 采用 $\mathrm{CuI} / \mathrm{DMEDA}$ 体系催化芳基溴代物 的羟基化时，需通过微波加热到 $180{ }^{\circ} \mathrm{C}$, 而且配体用量 高达 $50 \mathrm{~mol} \%$. 最近蒋晟小组 ${ }^{[6]}$ 发现 8 -羟基哇啉- $N$-氧化 物能有效地促进芳基溴代物甚至是氯代物的羟基化，尽 管仍然需要使用较大量的配体, 但是能催化不活泼的芳 基氯代物的转化是非常有意义的. 陈军民小组 ${ }^{[10]}$ 报道 了聚乙二醇为溶剂时, 采用 $10 \mathrm{~mol} \%$ 的 $\mathrm{CuI}$ 和 6.0 equiv. $\mathrm{KOH}$ 可促使芳基溴代物在较短时间内完全转化. 我们 则发现低负载的 $\mathrm{CuI}(2 \mathrm{~mol} \%) / 5$-溴-2-(1H-2-咪唑基)吡 啶 (4 mol\%)催化体系也能高效地催化芳基溴代物与 $\mathrm{CsOH}$ 或 $\mathrm{KOH}$ 的偶联反应得到取代苯酚 ${ }^{[1]}$. 在此研究 基础上，本文根据有关 2-芳胺基取代的 2-氧代乙酸能与 铜离子有效配位的报道 ${ }^{[12]}$, 考察了以这类羧酸为配体

\footnotetext{
* E-mail: jiangyw@mail.sioc.ac.cn \& yehao7171@163.com

Received June 1, 2012; revised June 15, 2012; published online June 20, 2012.

Project supported by the National Natural Science Foundation of China (No. 20921091).

国家自然科学基金(No. 20921091)资助项目.
} 
的铜催化的芳基溴代物的羟基化反应，发现碘化亚铜 2-(2,6-二甲基苯胺基)-2-氧代乙酸 (DMPAO) ${ }^{[20]}$ 可在低 催化剂负载 $(2 \mathrm{~mol} \% \mathrm{CuI}$ ) 的条件下促进该反应高效地 合成取代苯酚. 该羧酸配体可由 2,6-二甲基苯胺与氯代 乙醛酸乙酯反应后经水解以几乎定量的收率制备获 得 ${ }^{[13]}$, 来源十分方便.

\section{1 结果与讨论}

首先我们以 4-溴苯甲醚的差基化反应为模版, 进行 反应条件的优化. 先就 2-芳胺基取代的 2-氧代乙酸 (L1 L5) 对反应的促进作用进行了研究(表 1, Entries $1 \sim 5)$. 当以 $\mathrm{CuI} / \mathrm{DMPAO}$ 为催化体系, $\mathrm{DMSO} / \mathrm{H}_{2} \mathrm{O}(V$ : $V=1: 1$ )为混合溶剂, $\mathrm{KOH}$ 作碱, 催化 4-溴苯甲醚羟基 化时, 我们以 $86 \%$ 的收率分离得到 4-甲氧基苯酚 $\mathbf{2 a}$ (表 1, Entry 1). 其他几个 2-苯胺基-2-氧代乙酸类衍生物 $\mathbf{L 2} \mathbf{L 5}$ 作为配体反应收率都有所下降(表 1, Entry 1 vs. Entries 2 5). CuI 的用量降到 $2 \mathrm{~mol} \%$ 时, 与 DMPAO 的 组合依然具有很好的催化效率(表 1, Entry 6). 继续降低 $\mathrm{CuI}$ 用量发现底物的转化率明显下降(表 1, Entry 7). 其 它一价铜盐(如 $\mathrm{CuBr}, \mathrm{CuCl}, \mathrm{Cu}_{2} \mathrm{O}$ ) 证实亦可催化此反应
并获得较好收率(表 1, Entries 8～10). 用 $\mathrm{NaOH}$ 或 $\mathrm{CsOH} \cdot \mathrm{H}_{2} \mathrm{O}$ 取代 $\mathrm{KOH}$, 产率则有不同程度的下降(表 1, Entries $11 \sim 12$ ). 降低反应温度至 $100{ }^{\circ} \mathrm{C}$ 时, 产率也有 一定下降(表 1, Entry 13). 有趣的是, 当用我们之前报道 过的混合溶剂体系 DMSO- $t-\mathrm{BuOH}-\mathrm{H}_{2} \mathrm{O}(V: V: V=1$ : $1: 0.1)^{\text {时 }}{ }^{[3,11]}$, 4-甲氧基苯酚的产率只有 $36 \%$, 同时以 60\%的产率分离到双(4-甲氧基二苯基)醚 3a(表 1, Entry 14). 而提高混合溶剂体系中水的比例至 $(V: V: V=1$ : 1：1)时即可高收率的得到 4-甲氧基苯酚(表 1, Entry 15). 综合考虑温度和溶剂体系对反应的影响后，该羟基化反 应最优化条件定为: CuI $2 \mathrm{~mol} \%, \mathbf{L 1} 4 \mathrm{~mol} \%, \mathrm{KOH} 3$ equiv., $120{ }^{\circ} \mathrm{C}, \mathrm{DMSO} / \mathrm{H}_{2} \mathrm{O}(V: V=1: 1)$ 或 $t-\mathrm{BuOH} /$ $\mathrm{DMSO} / \mathrm{H}_{2} \mathrm{O}(V: V: V=1: 1: 1)$.

随后, 我们在优化的反应条件下对底物的适用范围 进行了考察(表 2). 如表 2 所示, 活性较高的芳基溴代物 (具有吸电子取代基)和活性比较低的芳基溴代物(具有 强给电子取代基)都能以较高的产率转化成相应的酚(表 2, Entries 1 14). 当反应底物是固体(如 4-苯基溴苯、硝 基溴苯等)时, 采用 $\mathrm{DMSO} / \mathrm{H}_{2} \mathrm{O}(V: V=1: 1)$ 混合溶剂 体系, 反应过程中底物会慢慢的凝结在反应管的顶端,

表 1 铜催化 4-溴苯甲醚差基化反应的配体和反应条件篎选 ${ }^{a}$

Table 1 Ligands and conditions screening for the copper-catalyzed hydroxylation of 4-bromoanisole

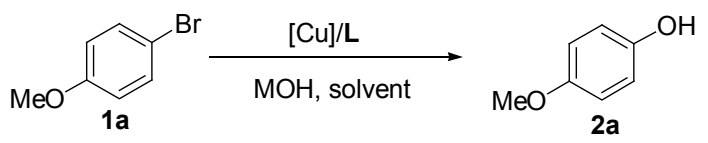

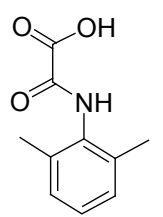

L1
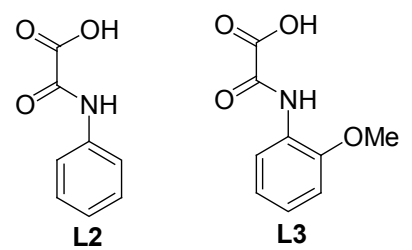

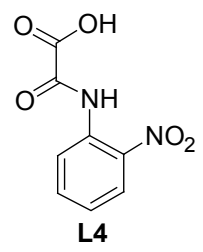

L4

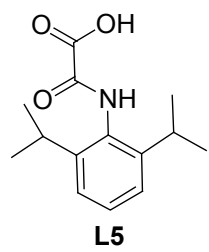

\begin{tabular}{cccccc}
\hline Entry & Cat. & Ligand & Cat. $/$ mol $\%$ & Base & Yield $/ \%$ \\
\hline 1 & $\mathrm{CuI}$ & $\mathbf{L 1}$ & 10 & $\mathrm{KOH}$ & 86 \\
2 & $\mathrm{CuI}$ & $\mathbf{L 2}$ & 10 & $\mathrm{KOH}$ & 35 \\
3 & $\mathrm{CuI}$ & $\mathbf{L 3}$ & 10 & $\mathrm{KOH}$ & 46 \\
4 & $\mathrm{CuI}$ & $\mathbf{L 4}$ & 10 & $\mathrm{KOH}$ & 43 \\
5 & $\mathrm{CuI}$ & $\mathbf{L 5}$ & 10 & $\mathrm{KOH}$ & 76 \\
6 & $\mathrm{CuI}$ & $\mathbf{L 1}$ & 2 & $\mathrm{KOH}$ & 90 \\
7 & $\mathrm{CuI}$ & $\mathbf{L 1}$ & 1 & $\mathrm{KOH}$ & $48\left(49^{c}\right)$ \\
8 & $\mathrm{CuBr}$ & $\mathbf{L 1}$ & 2 & $\mathrm{KOH}$ & 87 \\
9 & $\mathrm{CuCl}$ & $\mathbf{L 1}$ & 2 & $\mathrm{KOH}$ & 89 \\
10 & $\mathrm{Cu} \mathrm{O}^{b}$ & $\mathbf{L 1}$ & 2 & $\mathrm{NaOH}$ & 86 \\
11 & $\mathrm{CuI}$ & $\mathbf{L 1}$ & 2 & $\mathrm{CsOH} \cdot \mathrm{H}_{2} \mathrm{O}$ & 63 \\
12 & $\mathrm{CuI}$ & $\mathbf{L 1}$ & 2 & $\mathrm{KOH}$ & 85 \\
$13^{d}$ & $\mathrm{CuI}$ & $\mathrm{L1}$ & 10 & $\mathrm{KOH}$ & 80 \\
$14^{e}$ & $\mathrm{CuI}$ & $\mathrm{L1}$ & 2 & $\mathrm{KOH}$ & $38\left(60^{f}\right)$ \\
$15^{g}$ & $\mathrm{CuI}$ & $\mathbf{L 1}$ & 2 & 92 \\
\hline
\end{tabular}

${ }^{a}$ Reaction conditions: 4-bromoanisole $(1 \mathrm{mmol}), \mathrm{MOH}(3.0 \mathrm{mmol}),[\mathrm{Cu}] /$ ligand=1 : 2, DMSO $(1 \mathrm{~mL}), \mathrm{H}_{2} \mathrm{O}(1 \mathrm{~mL}), 120{ }^{\circ} \mathrm{C}, 24 \mathrm{~h} .{ }^{b}$ Isolated yield. ${ }^{c}$ Recovery of $1 \mathrm{a}$ ${ }^{d} 100{ }^{\circ} \mathrm{C} .{ }^{e} 1 \mathrm{~mL}$ of $t$-BuOH, $1 \mathrm{~mL}$ of DMSO and $0.1 \mathrm{~mL}$ of $\mathrm{H}_{2} \mathrm{O}$ were used. ${ }^{f} 4,4^{\prime}$-Methoxy diphenyl ether (3a) was isolated. ${ }^{g} 1 \mathrm{~mL}$ of $t$-BuOH, $1 \mathrm{~mL}$ of DMSO and $1 \mathrm{~mL}$ of $\mathrm{H}_{2} \mathrm{O}$ were used. 
表 $2 \mathrm{CuI} / \mathbf{L 1}$ 催化的芳基溴代物的羟基化 ${ }^{a}$

Table $2 \mathrm{CuI} / \mathbf{L 1}$ catalyzed hydroxylation of aryl bromides

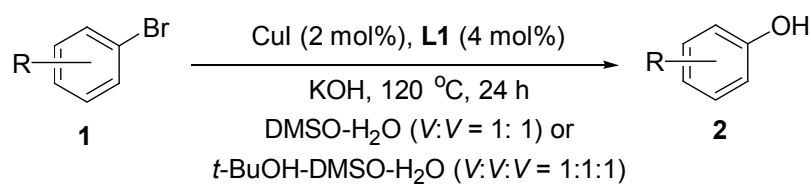

\begin{tabular}{|c|c|c|c|c|c|c|c|}
\hline Entry & Product & 2 & Yield $^{b} / \%$ & Entry & Product & 2 & Yield $^{b} / \%$ \\
\hline 1 & & $2 b$ & 83 & $8^{c, d}$ & & $2 \mathbf{i}$ & 75 \\
\hline 2 & & $2 c$ & 85 & $9^{c}$ & & $2 j$ & 81 \\
\hline 3 & & 2d & 87 & $10^{c}$ & & $2 k$ & 65 \\
\hline 4 & & $2 e$ & 82 & $11^{c}$ & & 21 & 87 \\
\hline $5^{c}$ & & $2 f$ & 87 & $12^{c, e}$ & & $2 m$ & 82 \\
\hline $6^{c}$ & & $2 \mathrm{~g}$ & 89 & $13^{c}$ & & $2 n$ & 61 \\
\hline $7^{c}$ & & $2 h$ & 95 & 14 & & 20 & 84 \\
\hline
\end{tabular}
yield. ${ }^{c} \mathrm{DMSO}-\mathrm{H}_{2} \mathrm{O}-t-\mathrm{BuOH}(V: V: V=1: 1: 1,8 \mathrm{~mL})$ were used. ${ }^{d} 100{ }^{\circ} \mathrm{C} .{ }^{e} 4$-Bromobenzonirile was used.

影响反应效果, 这一现象与我们之前的报道一致 ${ }^{[3]}$. 换 用 DMSO- $t-\mathrm{BuOH}-\mathrm{H}_{2} \mathrm{O}(V: V: V=1: 1: 1)$ 溶剂体系 可以很好的解决这一问题，同时反应的产率不受影响 (表 2, Entries 5～13). 多种官能团如甲基、甲硫基、硝基、 乙酰基、䍨基、酰胺基等在此反应条件下均能保持完整, 但是以 4-溴苯腈为底物羟基化后得到的是氧基水解后 的产物对差基苯甲酸(表 2, Entry 12). 该催化体系对杂 芳基溴代物如 2-溴代吡啶的羟基化同样有效(表 2, Entry 14).

在笁选条件时我们发现当减少三组分溶剂体系中 的水的含量时(表 1, Entry 14), 得到的主要产物为对称 二芳醚, 我们推测可能生成的苯酚在此反应体系中主要 以酚氧负离子形式存在, 该负离子在同样催化体系催化 下可进一步与芳基溴代物偶联得到对称二芳醚. 在此基 础上将碱换成 $\mathrm{Cs}_{2} \mathrm{CO}_{3}$ 时, 能以很好的产率一锅法合成 对称的芳香醚(图 1). 该反应体系没有采用强碱, 因此这 个反应的氧源应该来自体系中的水而不是强碱的氢氧
根. 这个结果与之前 Taillefer 等 ${ }^{[14]}$ 的报道相似.

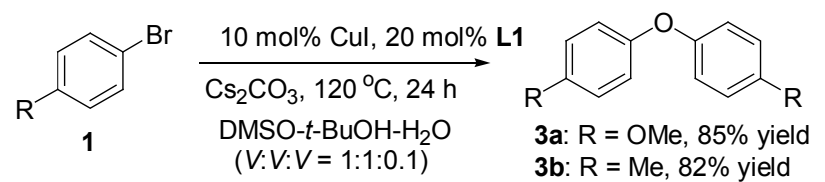

图 1 对称芳香醚的合成

Figure 1 Synthesis of symmetrical diaryl ether

\section{2 结论}

综上所述，我们发展了一个由碘化亚铜/2-(2,6-二甲 基苯胺基)-2-氧代乙酸组成的高效的催化体系, 并成功 用于芳基溴代物的差基化反应，催化剂的载量可低至 2 $\mathrm{mol} \%$ ，具有多种官能团耐受性，而且催化剂和配体都 价廉易得，具有较好的工业应用前景. 通过调整反应条 件，该催化体系还可用于促进芳香溴化物一锅法合成对 称的芳香醚. 


\section{3 实验部分}

\section{1 仪器与试剂}

核磁共振波谱由 Bruker AMX-300 或 Bruker AMX-400 型核磁共振仪测定(以 TMS 作内标); 质谱数 据由 HP-5989A 或者 SHIMADZU LCMS-2010 EV 型质 谱仪测定; 快速柱层析在硅胶 $\mathrm{H}$ 或 300 400 目硅胶上 进行, 所用石油醚为沸程 $60 \sim 90{ }^{\circ} \mathrm{C}$ 馏分. 薄层层析采 用 $\mathrm{GF}_{254}$ 高效硅胶板, 其它试剂均为市售分析纯.

\section{2 实验方法}

芳基溴代物羟基化的反应通用步骤: 在干燥的封管 内加入磁子, $12.0 \mathrm{mmol}$ 氢氧化钾, $0.08 \mathrm{mmol}$ 碘化亚铜, $0.16 \mathrm{mmol}$ 2-(2,6-二甲基苯胺基)-2-氧代乙酸, 抽真空并 充换氩气. 重复操作三次后, 再加入 $4.0 \mathrm{mmol}$ 芳基溴代 物, DMSO- $\mathrm{H}_{2} \mathrm{O}(V: V=1: 1,8 \mathrm{~mL})$ 或 DMSO- $\mathrm{H}_{2} \mathrm{O}-$ $t$-BuOH $(V: V: V=1: 1: 1,8 \mathrm{~mL})$. 用聚四氟乙烯塞 子封紧反应管, 放入预热好 $120{ }^{\circ} \mathrm{C}$ 的油浴内反应 $24 \mathrm{~h}$. 反应体系冷至室温, 用 $2 \mathrm{~mol} / \mathrm{L}$ 的盐酸调至 $\mathrm{pH}=1 \sim 2$, 用乙酸乙酯萃取, 饱和食盐水洗涤, 合并有机相并用无 水硫酸钠干燥, 旋转蒸发除去溶剂后, 快速柱层析得产 物.

合成对称二芳梄的通用步骤: 向干燥的反应管内分 别加入 $\mathrm{CuI}(19 \mathrm{mg}, 0.1 \mathrm{mmol}), \mathbf{L} 1(39 \mathrm{mg}, 0.2 \mathrm{mmol}$ )和 $\mathrm{Cs}_{2} \mathrm{CO}_{3}(978 \mathrm{mg}, 3 \mathrm{mmol})$. 抽真空并充换氩气, 重复操 作三次后, 加入芳基溴代物 (2 mmol)和 DMSO- $\mathrm{H}_{2} \mathrm{O}-t$ $\mathrm{BuOH}(V: V: V=1: 1: 0.1,4 \mathrm{~mL}) .120{ }^{\circ} \mathrm{C}$ 搅拌反应直 至 TLC 显示原料已完全消失. 反应体系冷至室温加入 水并用乙酸乙酯萃取, 合并有机相后用饱和食盐水洗 涤, 无水 $\mathrm{Na}_{2} \mathrm{SO}_{4}$ 干燥. 旋转蒸发除去溶剂后, 快速柱层 析分离提纯得相应产物.

\section{3 产物光谱数据}

4-甲氧基苯酚(2a) $)^{[1]}$ : ${ }^{1} \mathrm{H} \mathrm{NMR}\left(400 \mathrm{MHz}, \mathrm{CDCl}_{3}\right.$ ) $\delta$ : $6.80 \sim 6.75$ (m, 4H), 4.93 (brs, $1 \mathrm{H}), 3.76$ (s, 3H); ${ }^{13} \mathrm{C}$ NMR $\left(100 \mathrm{MHz}, \mathrm{CDCl}_{3}\right) \delta: 153.5,149.6,116.2(2 \mathrm{C}), 115.1$ (2C), 56.0; EI-MS $m / z: 124\left(\mathrm{M}^{+}\right)$.

苯酚 $(2 \mathbf{b})^{[2]}$ : ${ }^{1} \mathrm{H}$ NMR $\left(300 \mathrm{MHz}, \mathrm{CDCl}_{3}\right) \delta: 7.26 \sim$ $7.20(\mathrm{~m}, 2 \mathrm{H}), 6.96 \sim 6.90(\mathrm{~m}, 1 \mathrm{H}), 6.85 \sim 6.81(\mathrm{~m}, 2 \mathrm{H})$, $5.27(\mathrm{~s}, 1 \mathrm{H}) ;{ }^{13} \mathrm{C} \mathrm{NMR}\left(100 \mathrm{MHz}, \mathrm{CDCl}_{3}\right) \delta: 155.2,129.9$ (2C), 121.1, 115.5 (2C); EI-MS $m / z$ : $94\left(\mathrm{M}^{+}\right)$.

对甲基苯酚(2c) $)^{[2]}$ : ${ }^{1} \mathrm{H}$ NMR $\left(300 \mathrm{MHz}, \mathrm{CDCl}_{3}\right) \delta$ : 7.07 (d, $J=7.8 \mathrm{~Hz}, 2 \mathrm{H}), 6.79 \sim 6.76(\mathrm{~m}, 2 \mathrm{H}), 5.46$ (brs, $1 \mathrm{H}), 2.31(\mathrm{~s}, 3 \mathrm{H}) ;{ }^{13} \mathrm{C} \mathrm{NMR}\left(100 \mathrm{MHz}, \mathrm{CDCl}_{3}\right) \delta: 152.9$, 130.34, 130.32 (2C), 115.4 (2C), 20.5; EI-MS $\mathrm{m} / z: 108$ $\left(\mathrm{M}^{+}\right)$.

间甲基苯酚(2d) ${ }^{[15]}:{ }^{1} \mathrm{H}$ NMR $\left(400 \mathrm{MHz}, \mathrm{CDCl}_{3}\right) \delta$ :
$7.13 \sim 7.09$ (t, $J=7.2 \mathrm{~Hz}, 2 \mathrm{H}), 6.75 \sim 6.73(\mathrm{~d}, J=7.2 \mathrm{~Hz}$, $1 \mathrm{H}), 6.65 \sim 6.61(\mathrm{~m}, 2 \mathrm{H}), 4.78(\mathrm{~s}, 1 \mathrm{H}), 2.30(\mathrm{~s}, 3 \mathrm{H}) ;{ }^{13} \mathrm{C}$ NMR $\left(100 \mathrm{MHz}, \mathrm{CDCl}_{3}\right) \delta: 155.4,139.8,129.4,121.6$, 116.0, 112.2, 21.3; EI-MS $m / z$ : $108\left(\mathrm{M}^{+}\right)$.

4-(甲硫基)苯酚(2e $)^{[16]: ~}{ }^{1} \mathrm{H}$ NMR $\left(400 \mathrm{MHz}, \mathrm{CD}_{3} \mathrm{OD}\right)$ $\delta: 7.21 \sim 7.18(\mathrm{~m}, 2 \mathrm{H}), 6.77 \sim 6.73(\mathrm{~m}, 2 \mathrm{H}), 4.88$ (brs, $1 \mathrm{H})$, 2.39 (s, 3H); ${ }^{13} \mathrm{C}$ NMR (100 MHz, $\left.\mathrm{CD}_{3} \mathrm{OD}\right) \delta: 157.3,131.9$ (2C), 128.6, 117.0 (2C), 18.5; EI-MS m/z: $140\left(\mathrm{M}^{+}\right)$.

4-苯基苯酚 (2f $)^{[17]}$ : ${ }^{1} \mathrm{H}$ NMR $\left(400 \mathrm{MHz}, \mathrm{CD}_{3} \mathrm{OD}\right) \delta$ : $7.54 \sim 7.51(\mathrm{~m}, 2 \mathrm{H}), 7.47 \sim 7.43(\mathrm{~m}, 2 \mathrm{H}), 7.39 \sim 7.35(\mathrm{~m}$, $2 \mathrm{H}), 7.27 \sim 7.23(\mathrm{~m}, 1 \mathrm{H}), 6.89 \sim 6.85(\mathrm{~m}, 2 \mathrm{H}), 4.89(\mathrm{~s}$, $1 \mathrm{H}) ;{ }^{13} \mathrm{C}$ NMR (100 MHz, $\left.\mathrm{CD}_{3} \mathrm{OD}\right) \delta: 158.1,142.4,133.9$, 129.7 (2C), 129.1 (2C), 127.48 (2C), 127.45, 116.6 (2C); EI-MS $m / z: 170\left(\mathrm{M}^{+}\right)$.

2-萗酚(2g) $)^{[1]}:{ }^{1} \mathrm{H} \mathrm{NMR}\left(300 \mathrm{MHz}, \mathrm{CDCl}_{3}\right) \delta: 7.77 \sim$ $7.73(\mathrm{~m}, 2 \mathrm{H}), 7.68 \sim 7.65(\mathrm{~m}, 1 \mathrm{H}), 7.45 \sim 7.42(\mathrm{~m}, 1 \mathrm{H})$, $7.35 \sim 7.29(\mathrm{~m}, 1 \mathrm{H}), 7.13 \sim 7.07(\mathrm{~m}, 2 \mathrm{H}), 5.11(\mathrm{~s}, 1 \mathrm{H}) ;{ }^{13} \mathrm{C}$ NMR $\left(100 \mathrm{MHz}, \mathrm{CDCl}_{3}\right) \delta: 153.3,134.6,129.9,129.0$, 127.8, 126.6, 126.4, 123.7, 117.7, 109.5; EI-MS m/z: 144 $\left(\mathrm{M}^{+}\right)$.

3-硝基苯酚(2h) ${ }^{[1]}$ : ${ }^{1} \mathrm{H}$ NMR $\left(400 \mathrm{MHz}, \mathrm{CDCl}_{3}\right) \delta$ : $7.82 \sim 7.80(\mathrm{~m}, 1 \mathrm{H}), 7.71 \sim 7.70(\mathrm{t}, J=2.4 \mathrm{~Hz}, 1 \mathrm{H}), 7.43 \sim$ 7.39 (t, $J=4.4 \mathrm{~Hz}, 1 \mathrm{H}), 7.21 \sim 7.18(\mathrm{~m}, 1 \mathrm{H}), 5.73$ (brs, $1 \mathrm{H}) ;{ }^{13} \mathrm{C}$ NMR $\left(100 \mathrm{MHz}, \mathrm{CDCl}_{3}\right) \delta: 156.3,149.1,130.3$, 122.0, 115.9, 110.5; EI-MS $m / z: 139\left(\mathrm{M}^{+}\right)$.

2-硝基苯酚 (2i) ${ }^{[7]}$ : ${ }^{1} \mathrm{H}$ NMR $\left(300 \mathrm{MHz}, \mathrm{CDCl}_{3}\right) \delta$ : $10.60(\mathrm{~s}, 2 \mathrm{H}), 8.13 \sim 8.10(\mathrm{~m}, 1 \mathrm{H}), 7.62 \sim 7.56(\mathrm{~m}, 1 \mathrm{H})$, $7.18 \sim 7.15(\mathrm{~m}, 1 \mathrm{H}), 7.03 \sim 6.97(\mathrm{~m}, 1 \mathrm{H}) ;{ }^{13} \mathrm{C}$ NMR $(100$ $\left.\mathrm{MHz}, \mathrm{CDCl}_{3}\right) \delta: 153.1,137.5,133.6,125.0,120.2,119.6$; EI-MS $m / z: 139\left(\mathrm{M}^{+}\right)$.

4'羟基苯乙酮 $(\mathbf{2 j})^{[2]}:{ }^{1} \mathrm{H} \mathrm{NMR}\left(300 \mathrm{MHz}, \mathrm{CDCl}_{3}\right) \delta$ : $8.23(\mathrm{~s}, 1 \mathrm{H}), 7.94 \sim 7.91(\mathrm{~m}, 2 \mathrm{H}), 6.98 \sim 6.95(\mathrm{~m}, 2 \mathrm{H})$, $2.60(\mathrm{~s}, 3 \mathrm{H}) ;{ }^{13} \mathrm{C}$ NMR $\left(100 \mathrm{MHz}, \mathrm{CDCl}_{3}\right) \delta: 199.4,161.9$, 131.4 (2C), 129.2, 115.7 (2C), 26.3; EI-MS $m / z: 136\left(\mathrm{M}^{+}\right)$.

3 '-差基苯乙酮 $(\mathbf{2 k})^{[15]}$ : ${ }^{1} \mathrm{H} \mathrm{NMR}\left(400 \mathrm{MHz}, \mathrm{CDCl}_{3}\right) \delta$ : $7.55 \sim 7.54(\mathrm{~m}, 1 \mathrm{H}), 7.51 \sim 7.49(\mathrm{~m}, 1 \mathrm{H}), 7.34 \sim 7.31(\mathrm{t}$, $J=8.0 \mathrm{~Hz}, 1 \mathrm{H}), 7.20(\mathrm{br}, 1 \mathrm{H}), 7.14 \sim 7.11(\mathrm{~m}, 1 \mathrm{H}), 2.60(\mathrm{~s}$, 1H); ${ }^{13} \mathrm{C}$ NMR (100 MHz, $\left.\mathrm{CDCl}_{3}\right) \delta: 199.6,156.5,138.2$, 129.9, 121.0, 120.9, 141.7, 26.7; EI-MS $m / z: 136\left(\mathrm{M}^{+}\right)$.

4-氯苯酚 (2l) ${ }^{[2]: ~}{ }^{1} \mathrm{H}$ NMR $\left(300 \mathrm{MHz}, \mathrm{CDCl}_{3}\right) \delta: 7.18$ (d, $J=9.0 \mathrm{~Hz}, 2 \mathrm{H}), 7.76$ (d, $J=9.0 \mathrm{~Hz}, 2 \mathrm{H}), 5.29$ (s, 1H); ${ }^{13} \mathrm{C}$ NMR (100 MHz, $\mathrm{CDCl}_{3}$ ) $\delta: 153.4,129.8$ (2C), 126.3, 116.9 (2C); EI-MS m/z: $128\left(\mathrm{M}^{+}\right)$.

4-羟基苯甲酸 $(\mathbf{2 m})^{[4]}$ : ${ }^{1} \mathrm{H}$ NMR $(400 \mathrm{MHz}$, DMSO$\left.d_{6}\right) \delta: 12.4$ (brs, $\left.1 \mathrm{H}\right), 10.21$ (brs, $\left.1 \mathrm{H}\right), 7.81 \sim 7.78(\mathrm{~m}, 2 \mathrm{H})$, 
$6.84 \sim 6.81(\mathrm{~m}, 2 \mathrm{H}) ;{ }^{13} \mathrm{C}$ NMR $\left(100 \mathrm{MHz}, \mathrm{DMSO}-d_{6}\right) \delta$ : 167.1, 161.5, 131.5 (2C), 121.3, 115.0 (2C); EI-MS $m / z$ : $138\left(\mathrm{M}^{+}\right)$.

2-苯甲酰基氨基苯酚(2n) ${ }^{[18]}$ : ${ }^{1} \mathrm{H}$ NMR (400 MHz, $\left.\mathrm{CD}_{3} \mathrm{OD}\right) \delta: 7.97 \sim 7.95(\mathrm{~m}, 2 \mathrm{H}), 6.85 \sim 6.83(\mathrm{~m}, 1 \mathrm{H})$, $7.63 \sim 7.58(\mathrm{~m}, 1 \mathrm{H}), 7.55 \sim 7.51(\mathrm{~m}, 2 \mathrm{H}), 7.09 \sim 7.05(\mathrm{~m}$, $1 \mathrm{H}), 6.95 \sim 6.87(\mathrm{~m}, 2 \mathrm{H}), 4.88(\mathrm{~s}, 2 \mathrm{H}) ;{ }^{13} \mathrm{C}$ NMR $(100$ $\left.\mathrm{MHz}, \mathrm{CD}_{3} \mathrm{OD}\right) \delta$ : 168.4, 150.0, 135.8, 133.6, 129.8 (2C), 128.5 (2C), 127.2, 127.0, 124.0, 120.0, 117.0; EI-MS m/z: $213\left(\mathrm{M}^{+}\right)$.

2-差基吡啶 (2o $)^{[19]}$ : ${ }^{1} \mathrm{H}$ NMR $\left(300 \mathrm{MHz}, \mathrm{CDCl}_{3}\right) \delta$ : $13.69(\mathrm{~s}, 1 \mathrm{H}), 7.52 \sim 7.46(\mathrm{~m}, 1 \mathrm{H}), 7.14(\mathrm{dd}, J=6.3,1.2$ $\mathrm{Hz}, 1 \mathrm{H}), 6.03(\mathrm{~d}, J=9.3 \mathrm{~Hz}, 1 \mathrm{H}), 6.31(\mathrm{td}, J=6.6,0.9 \mathrm{~Hz}$, $1 \mathrm{H}) ;{ }^{13} \mathrm{C}$ NMR (100 MHz, $\left.\mathrm{CDCl}_{3}\right) \delta: 165.4,141.7,134.7$, 120.1, 106.9; EI-MS m/z: $95\left(\mathrm{M}^{+}\right)$.

双(4-甲氧基二苯基)醚(3a) ${ }^{[14]}$ ： ${ }^{1} \mathrm{H}$ NMR (400 MHz, $\left.\mathrm{CDCl}_{3}\right) \delta: 6.93 \sim 6.89(\mathrm{~m}, 4 \mathrm{H}), 6.86 \sim 6.82(\mathrm{~m}, 4 \mathrm{H}), 3.78$ $(\mathrm{s}, 6 \mathrm{H}) ;{ }^{13} \mathrm{C} \mathrm{NMR}\left(100 \mathrm{MHz}, \mathrm{CDCl}_{3}\right) \delta: 155.4(2 \mathrm{C}), 151.6$ (2C), 119.5 (4C), 114.8 (4C), 55.6 (2C); EI-MS m/z: 230 $\left(\mathrm{M}^{+}\right)$.

双(4-甲基二苯基)醚(3b) ${ }^{[14]}$ : ${ }^{1} \mathrm{H}$ NMR (400 MHz, $\left.\mathrm{CDCl}_{3}\right) \delta: 7.11 \sim 7.09(\mathrm{~m}, 4 \mathrm{H}), 6.90 \sim 6.86(\mathrm{~m}, 4 \mathrm{H}), 2.31$ $(\mathrm{s}, 6 \mathrm{H}) ;{ }^{13} \mathrm{C} \mathrm{NMR}\left(100 \mathrm{MHz}, \mathrm{CDCl}_{3}\right) \delta: 155.3$ (2C), 132.4 (2C), 130.1 (4C), 118.6 (4C), 20.7 (2C); EI-MS m/z: 198 $\left(\mathrm{M}^{+}\right)$.

\section{References}

[1] Tlili, A.; Xia, N.; Monnier, F.; Taillefer, M. Angew. Chem.,
Int. Ed. 2009, 48, 8725 .

[2] Zhao, D.; Wu, N.; Zhang, S.; Xi, P.; Su, X.; Lan, J.; You, J. Angew. Chem., Int. Ed. 2009, 48, 8729.

[3] Maurer, S.; Liu, W.; Zhang, X.; Jiang, Y.; Ma, D. Synlett 2010, 976.

[4] Yang, D.; Fu, H. Chem. Eur. J. 2010, 16, 2366.

[5] Paul, R.; Ali, M. A.; Punniyamurthy, T. Synthesis 2010, 4268.

[6] Yang, K.; Li, Z.; Wang, Z.; Yao, Z.; Jiang, S. Org. Lett. 2011, 13, 4340.

[7] Jing, L.; Wei, J.; Zhou, L.; Huang, Z.; Li, Z.; Zhou, X. Chem. Commun. 2010, 46, 4767.

[8] Thakur, K.; Sekar, G. Chem. Сотmu. 2011, 47, 6692.

[9] Mehmood, A.; Leadbeater, N. Catal. Commun. 2010, $12,64$.

[10] Chen, J.; Yuan, T.; Hao, W.; Cai, M. Catal. Commun. 2011, 12, 1463-1465

[11] Jia, J.; Jiang, C.; Zhang, X.; Jiang, Y.; Ma, D. Tetrahedron Lett. 2011, 52, 5593.

[12] Ferrando-Soria, J.; Pardo, E.; Ruiz-García, R.; Cano, J.; Lloret, F.; Julve, M.; Journaux, Y.; Pasán, J.; Ruiz-Pérez, C. Chem. Eur. J. 2011, 17, 2176.

[13] Narumi, T.; Ochiai, C.; Yoshimura, K.; Harada, S.; Tanaka, T.; Nomura, W.; Arai, H.; Ozaki, Taro.; Ohashi, Nami.; Matsushita, Shuzo.; Tamamura, H. Bioorg. Med. Chem. Lett. 2010, 20, 5853

[14] Tlili, A.; Monnier, F.; Taillefer, M. Chem. Eur. J. 2010, 16, 12299.

[15] Chan, C.; Chen, Y.; Su, C.; Lin, H.; Lee, C. Eur. J. Org. Chem. 2011, 7288.

[16] Hosoi, K.; Kuriyama, Y.; Inagi, S.; Fuchigami, T. Chem. Commun. 2010, 46, 1284.

[17] Schmidt, B.; Hölter, F. Org. Biomol. Chem., 2011, 9, 4914.

[18] Matsushita, H.; Lee, S.; Joung, M.; Clapham, B.; Janda, K. Tetrahedron Lett. 2004, 45, 313.

[19] Toganoh, M.; Fujino, K.; Ikeda, S.; Furuta, H. Tetrahedron Lett. 2008, 49, 1488.

[20] Zhang, Y.; Yang, X.; Yao, Q.; Ma, D. Org. Lett. 2012, 14, 3056

(Zhao, X.) 